\title{
A Robust Digital Watermarking Algorithm for Three Dimensional Meshes
}

\author{
Geri Bo \\ City Institute Dalian University of Technology, Dalian, 116600, China \\ ececity@dlut.edu.cn
}

Keywords: Watermarking; DFT; Affine transformation; Robust

\begin{abstract}
First, the algorithm converts 3d models into an affine invariant space, in order to obtain rotation in-variance and scale in-variance of the model; then we transform the distance of the grid point to the center to a signal in frequency domain using one-dimensional DFT. We embed a watermark into the signal in frequency domain, and then transform it back to a digital signal in spatial domain. Finally, according to the value of the new spatial signal, we modify the coordinates of points on the grid to get the digital watermark grid. Experimental results show that the algorithm can resist similar mesh simplification, plus noise and shearing attacks, and has good robustness.
\end{abstract}

\section{Introduction}

With CAD, CAM, CAE and other advanced manufacturing technology and the development of virtual reality technology, and networks are widely used in these areas. Three-dimensional model as a type of digital products, there are also issues of copyright protection. Adding watermark information in the 3D model to effectively protect 3D model's copyright information has become a research direction.

This paper proposes a digital watermarking algorithm in spatial domain with high robustness and relatively simple calculations. First, we should transform the 3D model into geometric invariant space. Secondly, by using DFT transform technique to modify the distance from the center to the vertex of 3D model is to embed the watermark information. Finally, the embedded watermark geometric invariant space model changes back to the original geometric space. In this paper, a large number of experiments carried out quantitative validation, introduction of several common attacks robust experimental results show that the digital watermarking algorithm in this paper is invisible on the common attacks robust. In this paper, through a large number of experimental quantitative verification, introduce several common attack experiments in robustness, results show that the proposed watermarking embedding algorithm is invisible and robust against common attacks.

\section{The Basic Principle of the Algorithm}

According to different 3D model modeling method, the three-dimensional model of digital watermark can be basically divided into grid watermark, parametric surface watermark and body data watermark, etc. In all types of three-dimensional model watermarking technology, the grid watermark technology is the direction of most studied. Triangular mesh representation is the most common representation of three-dimensional model representation. Other three-dimensional model representation can be converted into the triangle mesh representation, therefore, to study the watermarking technology through the triangular mesh representation. Triangle mesh representation has some of the same or substantially the same global geometric features, such as the size of the 3D model, surface curvature and the distance of each point on the surface to the center etc. To make the watermark embedded in the 3D solid model, you must choose a global geometric feature as an embedded object. In this paper, the algorithm selects the global geometric features of the distance of the surface point to the center as an embedded object. 


\section{Preprocessing Affine Invariance of 3D Model}

Three-dimensional model consists of a vertex set $p=\left\{p_{i}\right\}$ and a collection of $\mathrm{C}$ which has linking relationships. Each vertex $p_{i}$ has three-dimensional coordinates, i.e., $p_{i}=\left(x_{i}, y_{i}, z_{i}\right)$. The purpose of 3D model transformation is to transfer three dimensional data to one dimension signal for embedding watermarking. The 3D model obtained by affine transformation with rotation, scale transformation and translation invariant features. Specific process is as follows:

(1) Calculating all vertexes' the center of mass. The center of mass is defined as follows:

$\mu=\frac{1}{\mathrm{k}} \sum_{\mathrm{i}=0}^{\mathrm{k}-1} p_{i}=\left(\mu_{x}, \mu_{y}, \mu_{z}\right)$

(2) Model translation. $p_{i}=\left(x_{i}, y_{i}, z_{i}\right)$ obtained by subtracting the center of mass:

$$
p_{i}^{\prime}=\left(x_{i}^{\prime}, y_{i}^{\prime}, z_{i}^{\prime}\right)=\left(x_{i}-\mu_{x}, y_{i}-\mu_{y}, z_{i}-\mu_{z}\right)
$$

As a result, the new vertex coordinates are translation invariant.

(3) Principal component calculation [1]. The main ingredient is a vertex feature vector $T$, and the feature vector corresponds to the largest eigenvalue of the co-variance matrix of vertex coordinates. Among them, the co-variance matrix represented by the following formula:

$$
H=\left[\begin{array}{lll}
\sum_{i=0}^{k-1} x_{i}^{\prime 2} & \sum_{i=0}^{k-1} y_{i}^{\prime} x_{i}^{\prime} & \sum_{i=0}^{k-1} z_{i}^{\prime} x_{i}^{\prime} \\
\sum_{i=0}^{k-1} x_{i}^{\prime} y_{i}^{\prime} & \sum_{i=0}^{k-1} y_{i}^{\prime 2} & \sum_{i=0}^{k-1} z_{i}^{\prime} y_{i}^{\prime} \\
\sum_{i=0}^{k-1} x_{i}^{\prime} y_{i}^{\prime} & \sum_{i=0}^{k-1} y_{i}^{\prime} z_{i}^{\prime} & \sum_{i=0}^{k-1} z_{i}^{\prime 2}
\end{array}\right]
$$

(4) Model rotation. Rotate the model so that a feature vector T coincides with the z-axis, in order to achieve rotation in-variance.

\section{The Watermark Embedded}

Assuming that the original mesh model is $M_{0}=\left\{V_{0}, C_{0}\right\}, M_{0}$ composed of the vertex set $V_{0}$ and a collection of $C_{0}$ which has a link relationship, and the set contains $\mathrm{N}$ vertexes, and every coordinate value of vertex is determined by $\left(x_{0 i}, y_{0 i}, z_{0 i}\right)$, that is, $V_{0}=\left\{v_{0 i}\right\}, i=0,1, \ldots, N-1$. And the collection $C_{0}$ represents $C_{0}=\left\{\left\{i_{k}, j_{k}\right\}\right\}, k=0,1, \ldots, m-1,0 \leq i_{k} \leq N-1,0 \leq j_{k} \leq N-1$.Specifically watermark embedding process is as follows:

(1) Pre-treatment of the model.

(2) Input the watermark information to be embedded or the key $\mathrm{K}$ into the pseudo random sequence generator, and generate the corresponding binary watermark sequence $W=\left\{w_{l}\right\}, N_{w}$ is the length of the watermark sequence.

(3) Rowing the grid vertex into a one-dimensional sequence of vertexes, and transform into spherical $x_{i}, y_{i}, z_{i}$ coordinates $\theta_{i}, \varphi_{i}, \gamma_{i}$, the relationship between them is shown in Figure 1.

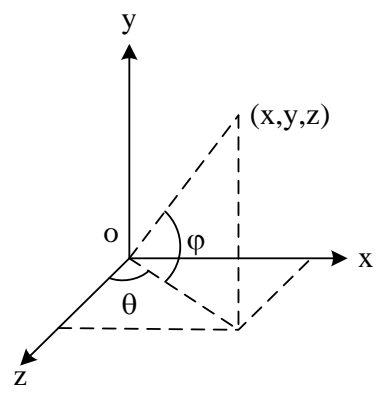

Fig.1. Relationship between $x, y, z$ and $r, \theta, \varphi$ 


$$
\begin{aligned}
& \mathrm{r}_{i}=\sqrt{x_{i}^{2}+y_{i}^{2}+z_{i}^{2}} \\
& \theta_{i}= \begin{cases}\arccos \left(z_{i} / \sqrt{x_{i}^{2}+z_{i}^{2}}\right), \quad x_{i} \geq 0 \\
2 \pi-\arccos \left(z_{i} / \sqrt{x_{i}^{2}+z_{i}^{2}}\right), \quad x_{i}<0\end{cases} \\
& \phi_{i}= \begin{cases}\arccos \left(\sqrt{x_{i}^{2}+z_{i}^{2}} / r_{i}\right), & y_{i} \geq 0 \\
-\arccos \left(\sqrt{x_{i}^{2}+z_{i}^{2}} / r_{i}\right), & y_{i}<0\end{cases}
\end{aligned}
$$

(4) $r_{i}$ Obtained in the previous step is arranged according to certain order, and order takes $S \times N_{w}$ elements, and is divided into $N_{w}$ groups, then each group contains $S$ elements, that is $R=\left\{r_{l j}\right\}, l=0,1, \ldots N_{w}, j=0,1, \ldots, \mathrm{S}-1$. Each group $r_{l j}$ is as an embedding unit to embed a watermark information. Embedding method as follows:

DFT transform is performed on a set of $r_{l j}$ to get the corresponding DFT coefficient $R_{l j}$. Using the formula (5), the $l$ bit of the watermark information $w_{l}$ is embedded into the DFT coefficients $R_{l j}$.

$$
\left|R_{l j}^{\mathrm{w}}\right|=\left|R_{l j}\right|+\alpha \varepsilon_{j} w_{l}
$$

Among them, $\alpha$ is embedding strength coefficient,and it controls the total energy of the embedded watermark signal; And $\varepsilon=\left(\varepsilon_{0}, \varepsilon_{1}, \cdots, \varepsilon_{j}\right)$ is $(0,1)$ uniformly distributed random numbers used for modulation. This is important to note that in the embedded watermark can't select dc component $R_{l 0}$ and coefficient $R_{s / 2}^{l}$. Then IDFT of $R_{l j}^{w}$ to get the $r_{l j}^{w}$ that contains the watermark information.

(5)Inverse sort on $r_{l j}^{w}$ to get $r_{i}^{w}$, Using equation (6) can be obtained watermarked middle transition vertex coordinates: :

$$
\begin{aligned}
\mathrm{x}_{i}^{\prime} & =r_{i}^{w} \times \cos \phi_{i} \times \sin \theta_{i} \\
y_{i}^{\prime} & =r_{i}^{w} \times \sin \phi_{i} \\
z_{i}^{\prime} & =r_{i}^{w} \times \cos \phi_{i} \times \cos \theta_{i}
\end{aligned}
$$

(6) Vertex coordinate $\left(\mathrm{x}_{i}^{\prime}, y_{i}^{\prime}, z_{i}^{\prime}\right)$ obtained above are pre-treated as 2.1 inverse transformation, then obtains the final watermarked vertexes $\left(x_{i}^{w}, y_{i}^{w}, z_{i}^{w}\right)$.

\section{The Watermark Extraction}

(1) Using model pre-processing, the original model $M_{o}$ and the detected model $M_{d}$ is of the same transformation respectively, after transformation vertex coordinates as: $v_{o i}=\left\{\left(x_{o i}, y_{o i}, z_{o i}\right)\right\}$, $v_{d i}=\left\{\left(x_{d i}, y_{d i}, z_{d i}\right)\right\}$.

(2) Because some of the attacks will change the original mesh representation, such as simplifying the attack, cropping attack and re-gridding etc. the watermark detection algorithm can't extract the watermark information directly from the attack model. In response to this situation, the use of re-sampling to restore the original mesh representation of model. Re-sampling process is as follows[2]: from the center of the original model elicits a ray to the vertex $v_{o i}$ and intersects with a three-dimensional model to be tested .If there is more than one intersection points $v_{d i}$ and which are the nearest intersection of $v_{o i}, v_{d i}$ and $v_{o i}$ is considered a matching point, otherwise take $v_{d i}=v_{o i}$.

(3) Using the previous coordinate conversion formula (4), $\left(x_{o i}, y_{o i}, z_{o i}\right),\left(x_{d i}, y_{d i}, z_{d i}\right)$ are respectively converted to $\left(r_{o i}, \varphi_{o i}, \theta_{o i}\right),\left(r_{d i}, \varphi_{d i}, \theta_{d i}\right)$. Similarly, the obtained $r_{o i}, r_{d i}$ are sorted and 
grouped in the same order of watermark embedding, namely: $R_{o l}=\left\{r_{o l j}\right\}, R_{d l}=\left\{r_{d l j}\right\}, l=0,1, \cdots, N_{W}$; $j=0,1, \cdots, S-1$.

(4) Calculating a difference between each set of the DFT coefficients $R_{d l j}$ and $R_{o l j}$ :

$$
\Delta_{l j}=R_{d l j}-R_{\text {olj }}
$$

(5) Using formula (8) to extract the watermark sequence:

$$
W_{l}^{d}=\operatorname{sign}\left(\sum_{j=0}^{s-1} \Delta_{l j}\right) \quad l=0,1, \ldots, \mathrm{N}_{\mathrm{w}}-1
$$

(6)Correlation calculation . The correlation coefficient $C_{\text {or }}$ is defined as:

$$
\operatorname{Cor}=\frac{\sum_{i=0}^{N_{w}-1}\left(w_{i}^{d}-\bar{W}^{d}\right)\left(w_{i}-\bar{W}\right)}{\sqrt{\sum_{i=0}^{N_{w}-1}\left(w_{i}^{d}-\bar{W}^{d}\right)^{2}\left(w_{i}-\bar{W}\right)^{2}}}
$$

$W^{d}$ of equation (9)is extracted watermark sequence, $W$ is the original watermark sequence , $\bar{W}^{d}$ is the average of the various in the sequence $W^{d}, \bar{W}$ is the average of the sequence $W$ of each one , $N_{w}$ is the length of the watermark sequence.

\section{The Experimental Results}

In order to verify the algorithm in this paper, this experiment uses a rabbit 3D model to test, the model consists of 1751 vertexes and 2999 triangular faces.

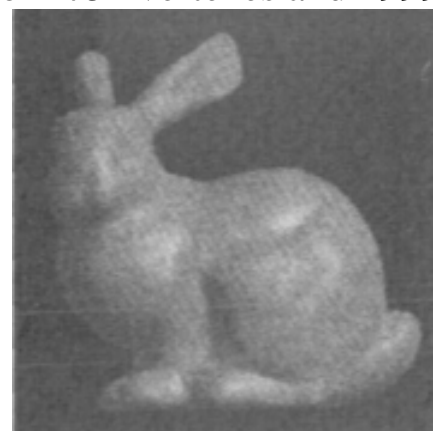

Fig.2. Original model

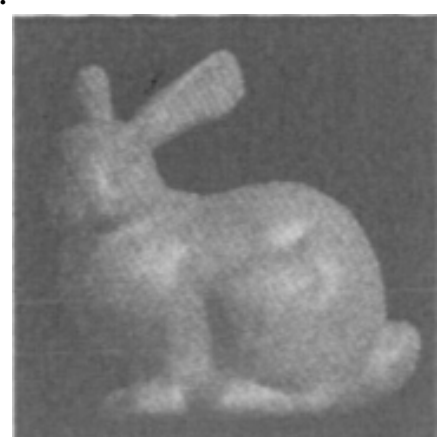

Fig.3. Add watermarks model

In the experiment, the original watermark sequence length $N_{w}$ is 32, the number of vertexes $S$ of each set is 48, the embedding strength $A$ is 0.04 . Figure 2 and Figure3 are the original 3D model and the embedded watermark model. Comparing Figure 2 and Figure 3 show that the watermarking algorithm satisfies the conditions of watermark invisibility. Testing the robustness of the algorithm, respectively tested it with simplified attack, noise attack and shear attacks.

When testing the ability of this algorithm to resist the simplify attack, taking the StanMelax algorithm to simplify the 3D model of embedded watermark, the experimental results are shown in Table 1 and Figure 4. As can be seen from Table 1, even if the attack model has only a few vertexes, the algorithm still showed strong robustness.

Tab.1.The results of simplifies attack

\begin{tabular}{c|c|c|c}
\hline The remaining number of vertexes & 1178 & 785 & 392 \\
\hline Simplified rate & $25 \%$ & $50 \%$ & $75 \%$ \\
\hline Cor & 1.0 & 0.9393 & 0.6888 \\
\hline
\end{tabular}




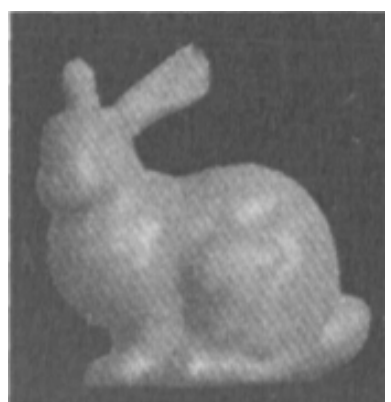

(a) $25 \%$

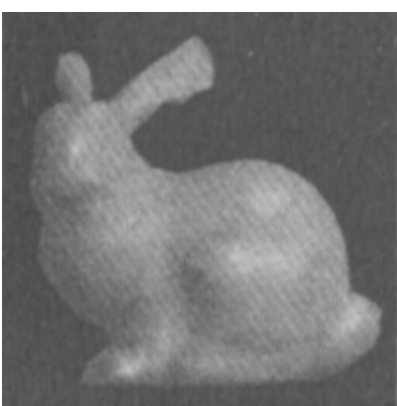

(b) $50 \%$

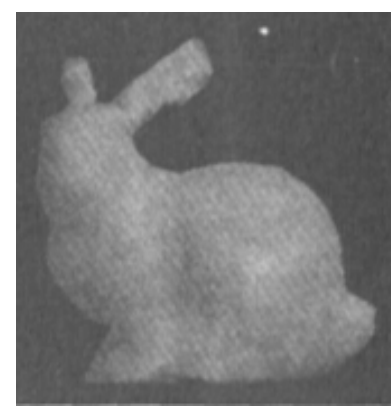

(c) $75 \%$

Fig.4. Simplifies attack

Noise attack experiments, added uniform noise to the vertex coordinates that have been added the watermark model. Joins a noise vector to each vertex of model that have been added the watermark. Noise vector direction along the grid vertex to the origin of attachment, the magnitude of the noise is defined as the ratio of the length of the noise vector and the distance of the grid vertex to the grid center, which reflects the strength of the noise. Noise attack experiment results are shown in Table 2, mesh model added noise is shown in Figure 5.

Tab.2. The results of noise attack

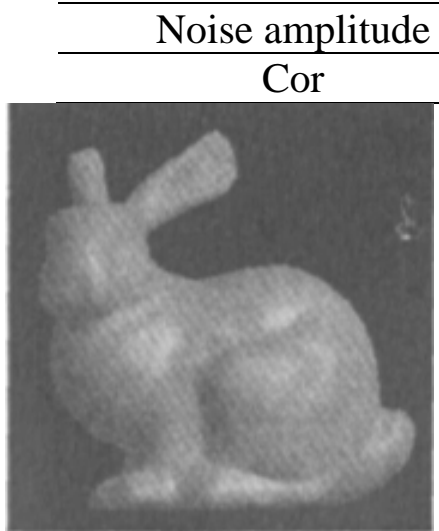

(a) 0.01

\begin{tabular}{r|r|r} 
& 0.005 & 0.01 \\
\hline & 0.9393 & 0.875 \\
& & \\
&
\end{tabular}

(b) 0.015

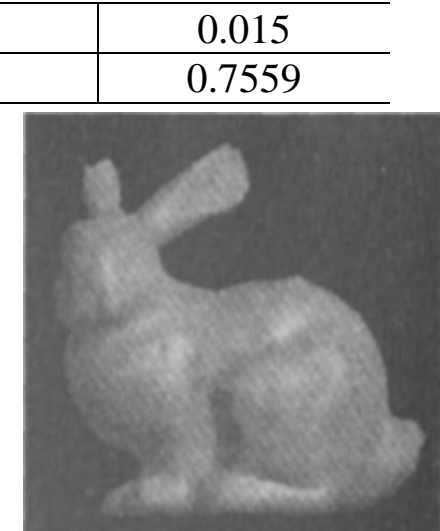

(c) 0.02

Fig.5. Noise attack

This article from the five kinds of different situations shear test. After shearing, the rest of the vertexes respectively change to $90 \%, 75 \%, 50 \%, 25 \%$ and $15 \%$ of the original model, and the results are shown in Figure 6 and Table 3. Table 3 shows that the algorithm has strong robustness to shear attack.

Tab.3. The results of share attack

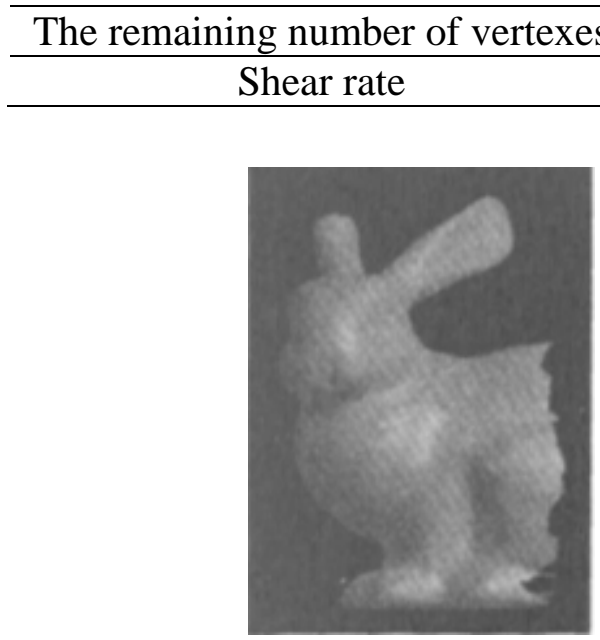

(a) $25 \%$

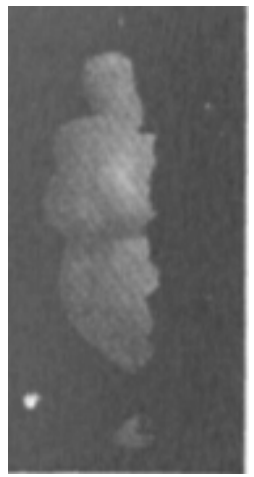

(b) $75 \%$

\begin{tabular}{l|l}
\hline 1178 & 785 \\
\hline $25 \%$ & $50 \%$ \\
\hline
\end{tabular}

Fig.6. Share attack

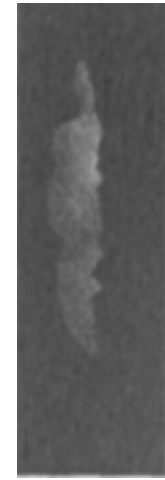

(c) $85 \%$ 


\section{Conclusion}

This paper presents a robust $3 \mathrm{~d}$ mesh model digital watermarking algorithm, and the algorithm achieves the DFT transform for vector length of model center to vertex, then realize of watermark embedding by modifying the modulus value of DFF coefficients. Simulation results show that embedded watermark is invisible, and can resist the general 3d mesh models attacks, such as mesh simplification attack, shear attack, noise attack, etc.

\section{References}

[1] A. Tefas, A. Kallivas and I. Potas. Watermarking of 3D Models Using Principal Component Analysis[J].IEEE International Conference on Acoustics, Speech, and Signal Processing(ICASSP’0 3),April 6-10,2003,5:676-679.

[2] H H S Ip, Z Q Yu, L F Kowk. A Robust Watermarking Scheme for 3D Triangular Mesh Models[J].Pattern Recognition,2003,36(11):2603-2614.

[3] Stan Melax. A imple, fast, and effective polygon reduction algorithm[J].Game Developer,1998,11:44-49.

[4] Zhang Jing, Zheng Guoqin. Three-dimensional network of digital watermarking algorithm based on geometric features[J].Computer Aided Design and Computer Graphics,2005,17(14):740-747. 